\title{
Associação das Medidas Antropométricas de Localização de Gordura Central com os Componentes da Síndrome Metabólica em uma Amostra Probabilística de Adolescentes de Escolas Públicas
}

\begin{abstract}
RESUMO
Avaliou-se a associação entre medidas antropométricas de localização de gordura central: circunferências da cintura (CC) e abdominal (CA) e as razões cintura/quadril (RCQ) e cintura/estatura (RCE), independente do percentual de gordura corporal (\%GC) e índice de massa corporal (IMC), com os componentes da síndrome metabólica (SM) em uma amostra probabilística de 610 (222 meninos e 388 meninas) adolescentes de 12 a 19 anos de escolas públicas de Niterói, RJ. Foram avaliados glicose e perfil lipídico pelo método enzimático automatizado, insulina pela técnica de radioimunoensaio,\%GC pela bioimpedância elétrica e a pressão arterial sistólica (PAS) e diastólica com manômetro digital. A associação entre as medidas antropométricas foi verificada por meio de regressão linear múltipla, com ajuste para IMC, \%GC e idade. Nos meninos, a associação positiva entre $C C(\beta=1,03, p<0,01)$ e $\operatorname{RCE}(\beta=2,33, p$ $<0,05 ; \beta=2,12$ e $p<0,01)$ com triglicerídios foi mantida independente de IMC e \% de GC, respectivamente. A CC se correlacionou com a PAS independente do $\% G C$, tanto nos meninos $(\beta=0,70 p<0,01)$ quanto nas meninas $(\beta=0,68$ $p<0,01)$. Conclui-se que a CC foi a medida de gordura central que apresentou a melhor associação com os componentes da SM nos adolescentes. (Arq Bras Endocrinol Metab 2008; 52/4:649-657)
\end{abstract}

Descritores: Adolescentes; Gordura abdominal; Síndrome metabólica; Risco cardiovascular.

\section{ABSTRACT}

Association Between Central Body Anthropometric Measures and Metabolic Syndrome Components in a Probabilistic Sample of Adolescents from Public Schools.

It was investigated the association of waist circumference (WC), abdominal circumference (AC), Waist-to-Hip ratio(WHR) and Waist-Height ratio (WHtR) adjusted by body mass index (BMI) and \% body fat (BF) with metabolic syndrome components in a probabilistic sample of 610 adolescents aged 12-19 years (222 boys and 388 girls) from public schools of Niterói, Rio de Janeiro. Fasting glucose and lipids were assayed using automated enzymatic method and insulin was determined by radioimmuno assay. Systolic (SBP) and diastolic (DBP) blood pressure were measured using an automated recorder and $\%$ BF by electric bioimpedance. The association of the anthropometric measures with metabolic syndrome components was evaluated by multivariate linear regression adjusted according to the age, BMI or \%BF. In boys, a positive association between WC $(\beta=1.03 p<0.01)$ and $\mathrm{WHtR}(\beta=2.33, p<0.05 ; \beta=2.12$ and $p<0.01)$ with triglycerides was maintained after $\mathrm{BMI}$ and \% BF adjusting, respectively. WC was associated with SBP after \% BF adjusting both for boys $(\beta=0.70 p<0.01)$ and girls $(\beta=0.68 p<0.01)$. In conclusion, WC was the measure of central body fat that presented the best association with components of metabolic syndrome in adolescents. (Arq Bras Endocrinol Metab 2008; 52/4:649-657)

Keywords: Adolescents; Abdominal fat; Metabolic syndrome; Cardiovascular risk. artigo original

\author{
MARLene M. Alvarez
}

Ana Carolina R. e Vieira

ROSELY SICHIERI

GLORIA V. DA VEIGA

Instituto de Nutrição Josué de Castro da Universidade Federal do Rio de Janeiro (UFRJ), RJ, Brasil (MMA, GVV); Hospital Universitário Antônio Pedro da Universidade Federal Fluminense (HUAP/UFF), Rio de Janeiro, RJ, Brasil (MMA); Instituto de Medicina Social da Universidade do Estado do Rio de Janeiro (UERJ), Rio de Janeiro, RJ, Brasil (ACRV, RS).

Recebido em 15/10/2007

Aceito em 03/04/2008 


\section{INTRODUÇÃO}

$\mathrm{O}$ ACÚMULO DE GORDURA CENTRAL está associado à presença de alterações metabólicas que indicam risco cardiovascular, como aumento da resistência à insulina, hipertrigliceridemia, baixo HDL-C (high density lipoprotein cholesterol) e alteração da pressão arterial, que são descritas como componentes da síndrome metabólica (SM), cujo aumento da prevalência tem sido observado em jovens obesos (1). No Brasil, a SM foi verificada em 3,2\% das meninas de 12 a 19 anos estudantes de escolas públicas de Niterói, RJ (21,4\% entre as com sobrepeso) (2) e em 26,1\% entre adolescentes obesos com história familiar de diabetes melito, atendidos em ambulatório na cidade de São Paulo (3).

Para avaliar a gordura corporal na região central, são utilizadas em estudos epidemiológicos medidas antropométricas, como circunferências da cintura (CC), razão cintura/quadril (RCQ), razão cintura/estatura (RCE), pela praticidade e pelo baixo custo (4), já que métodos mais sensíveis, como a tomografia computadorizada e a ressonância magnética (5), por causa do alto custo, são inviáveis nesse tipo de estudo.

O International Diabetes Federation (IDF) (6) elegeu a CC acima de valores específicos, de acordo com a etnia, como componente obrigatório para diagnóstico da SM em adultos. A proposta anterior da OMS-1998 (Organização Mundial da Saúde) (7) incluía valores elevados de RCQ entre os critérios de diagnóstico. A definição de qual dessas medidas mais bem se correlaciona com os demais componentes da SM ainda é controversa, com alguns autores indicando a CC $(8,9)$ e outros a RCQ $(10,11)$.

Em adolescentes, a questão parece ser ainda mais complexa. A RCQ pode ser uma medida inapropriada para esta faixa etária já que a largura pélvica modificase rapidamente durante o estirão do crescimento, e o índice pode estar refletindo mais essa variação do que, propriamente, o acúmulo de gordura (12). Entretanto, a RCQ apresentou maior capacidade preditiva para HDL-C tanto em adolescentes brasileiras com sobrepeso (13) quanto em mexicanas-americanas (14). Por outro lado, a CC foi considerada o melhor índice antropométrico para predição de fatores de risco para doença cardiovascular (DCV) em adolescentes americanos (15). Um outro indicador, sugerido recentemente, é a relação cintura/estatura, teoricamente um bom marcador para monitorar excesso de peso em jovens, por que considera o crescimento tanto da cintura quanto da estatura (4)
Além dos diferentes marcadores, diversas metodologias e nomenclaturas têm sido utilizadas para descrever as medidas antropométricas que estimam a gordura central, sobretudo a CC para a qual já foram citados 14 locais diferentes para sua aferição (16). A falta de padronização das medidas de circunferências dificulta a comparabilidade dos resultados dos estudos e limita o entendimento do desempenho de cada medida para identificação do risco, especialmente na fase da adolescência, quando os indicadores modificam-se acentuadamente.

A avaliação de risco cardiovascular por meio de medidas antropométricas de gordura central é ainda pouco investigada em adolescentes. Considerando que a instalação precoce dos fatores de risco cardiovascular aumenta os efeitos deletérios da doença na vida adulta, a identificação de medidas simples e não-invasivas que se associem com estes fatores em adolescentes saudáveis pode ser de grande utilidade para a prevenção das doenças cardiovasculares no futuro. O objetivo do estudo foi avaliar medidas antropométricas que estimem a localização de gordura central e sua associação com os componentes da SM em adolescentes, verificando se este efeito é independente do índice de massa corporal (IMC) e do percentual de gordura corporal (\%GC).

\section{MATERIAL E MÉTODOS}

O estudo foi desenvolvido em amostra probabilística de adolescentes de 12 a 19 anos, estudantes da rede de ensino estadual, da cidade de Niterói, Rio de Janeiro. Em 2001, ano-base para o cálculo da amostra, 25.102 alunos de 12 a 19 anos estavam matriculados nas 33 escolas da rede. O cálculo de amostra baseou-se na prevalência de hipercolesterolemia, estimada em 25\% (17), intervalo de confiança de 95\% (IC95\%), precisão absoluta de cinco pontos percentuais, e no desenho de amostragem por conglomerado (sorteio de turmas), perfazendo o total de 600 estudantes (18). Prevendose perda de $30 \%$, conforme estudo-piloto, a amostra foi estimada em 780 adolescentes.

Nas turmas sorteadas, 757 alunos atendiam aos critérios de elegibilidade (não ser portador de deficiência física que impedisse a avaliação antropométrica, não estar grávida ou amamentando, não ser portador de obesidade secundária a outras patologias ou estar em uso de medicamentos que interferissem no resultado dos exames). Só participaram do estudo os adolescentes que quiseram e apresentaram o termo de consentimen- 
to livre e esclarecido assinado pelo responsável ou por ele próprio, quando maior de 18 anos. Ao final totalizaram 610 adolescentes que foram avaliados por equipe treinada, sob supervisão da pesquisadora responsável pelo projeto, no período de junho a dezembro de 2003.

$O$ peso foi aferido em balança eletrônica, com capacidade até $150 \mathrm{~kg}$ e variação de $0,05 \mathrm{~kg}$ e a estatura, com o uso de antropômetro portátil, com extensão até $200 \mathrm{~cm}$ e variação de $0,1 \mathrm{~cm}$. Os estudantes estavam descalços, com roupas leves e em posição ortostática (19). Foram realizadas duas mensurações de estatura, admitindo-se variação máxima de $0,5 \mathrm{~cm}$, considerando a média para a análise, repetindo-se o procedimento no caso de ultrapassar essa variação.

A composição corporal foi estimada por meio da bioimpedância elétrica, utilizando-se o analisador de composição corporal Tanita TBF - 305 (sistema membro inferior a membro inferior). A densidade corporal foi estimada por meio de equação oferecida pelo próprio fabricante, específica para idade e sexo, e o \%GC foi estimado a partir da densidade, utilizando-se equações específicas para a idade (20).

Para a mensuração das circunferências, foi utilizada a metodologia do manual de padronização de medidas antropométricas (21). As circunferências da cintura e do quadril foram realizadas utilizando fita métrica inextensível de $200 \mathrm{~cm}$ e variação de $0,1 \mathrm{~cm}$, na menor circunferência do tronco e na circunferência máxima sobre as nádegas, respectivamente. A medida da CA foi realizada na cicatriz umbilical. Foram realizadas duas medidas, calculando-se a média e admitindo-se variação máxima de $1 \mathrm{~cm}$ entre as duas, repetindo-se o procedimento no caso de ultrapassar essa variação.

Calculou-se a RCQ (circunferência da cintura em $\mathrm{cm} /$ circunferência do quadril em $\mathrm{cm}$ ) e a RCE (circunferência da cintura em cm/estatura em cm) e o IMC = peso/estatura ${ }^{2}$. A aferição da pressão arterial sistólica (PAS) e diastólica (PAD) foi realizada com um manômetro digital da marca Pro Check, em duplicata, com intervalo de 2 minutos entre as duas medidas. Se a primeira das duas medidas diferisse em mais do que 15 $\mathrm{mmHg}$, uma leitura adicional era realizada, calculandose a média. Para a verificação da pressão arterial, os adolescentes foram orientados a ficarem 1 hora sem realização de atividade física, 30 minutos sem ingestão de café e sem uso de cigarro.

Para a avaliação bioquímica foi colhida amostra de, aproximadamente, $10 \mathrm{ml}$ de sangue, em dia consecutivo ao da avaliação antropométrica, com as adolescentes em jejum de 12 horas. Foram realizadas dosagens de glicose, HDL-C e triglicerídeos pelo método enzimático automatizado Extress Plus, em laboratório reconhecido pela Sociedade Brasileira de Patologia. A insulina plasmática foi analisada pela técnica de radioimunoensaio, no Laboratório de Fisiologia da Universidade do Estado do Rio de Janeiro (UERJ).

Foram obtidos o LDL-C (low-density lipoprotein cholesterol) (22) e o HOMA-IR (homeostatic model assessment - insulin resistance) (23) mediante fórmulas específicas.

A análise dos dados foi realizada por meio do programa Statistical Analysis System, versão 8.2. (SAS, Institute Inc, Cary, NC). Foram calculadas as médias das variáveis contínuas e seus respectivos intervalos de confiança (IC) de 95\%, sendo testadas diferenças significativas entre os grupos pelo valor de $\mathrm{p}<0,05$, obtido pela regressão linear.

Avaliou-se a associação entre as variáveis antropométricas de adiposidade central (independentes) e as variáveis bioquímicas, e a pressão arterial (dependentes) por meio da regressão linear bivariada e múltipla, ajustada pelas variáveis IMC ou \%GC e idade.

As variáveis insulina, $\mathrm{HDL}-\mathrm{C}$, triglicerídeos e $\mathrm{HO}$ MA-IR foram transformadas em logaritmos para análise de regressão linear, por não apresentarem distribuição normal verificada pelo teste Kolmogorov-Smirnov. Foi considerado $\mathrm{p}<0,05$ para significância estatística das variáveis no modelo. Para todas as análises levou-se em consideração a expansão da amostra e o efeito do desenho da amostra por conglomerado (turmas), utilizando-se o comando proc surveyreg do SAS.

O projeto da pesquisa foi aprovado pelo Comitê de Ética em Pesquisa da Faculdade de Medicina do Hospital Universitário Clementino Fraga Filho da Universidade Federal do Rio de Janeiro (HUCFF/UFRJ).

\section{RESULTADOS}

A partir dos 757 adolescentes elegíveis para a pesquisa, obtiveram-se dados antropométricos de 610 (43 não obtiveram autorização dos pais, 85 não quiseram participar e 19 não compareceram nos dias da coleta de dados). Para a avaliação bioquímica houve perda adicional de 33 adolescentes e para análise da insulina, 68 amostras de plasma ainda foram perdidas em virtude de problemas técnicos. 
Os meninos apresentaram médias significativamente maiores de CC e estatura, enquanto as meninas apresentaram quase o dobro do valor dos meninos, para média de \%GC. As médias de pressão arterial também foram significativamente mais elevadas para o sexo masculino, enquanto as meninas apresentaram valores mais altos para colesterol total e LDL-C (Tabela 1).

O IMC se associou de maneira positiva e significativa com todas as medidas de gordura central, embora a associação tenha sido mais forte para CC, CA e RCE, já que cerca de $78 \%$ a $85 \%$ da variação nestas medidas se dá em função da variação de IMC, enquanto para RCQ este percentual foi de apenas $13 \%$ a $23 \%$. A associação com o \%GC teve o mesmo comportamento, embora com poder de explicação menor que o IMC ( $\mathrm{R}^{2}$ variando de 0,42 a 0,78 e valores de $\beta$ menores). Para os meninos todas as medidas de gordura central se associaram de maneira positiva e significativa com triglicerídeos e com PAS, com exceção da RCQ com a PAS, embora o poder de explicação da variação destas variáveis tenha sido re- duzido ( $\mathrm{R}^{2}$ variando de 0,03 para $\mathrm{CC}$ com $\mathrm{PAD}$ a 0,18 para CC com PAS). Verificou-se, ainda, associação positiva e significativa entre a CC e a CA com a PAD. Para as meninas todas as medidas se associaram positivamente com a PAS e com a PAD (com exceção da RCQ para a PAD) e o poder de explicação na variação das medidas foi de $4 \%$ para a RCQ com a PAS a $22 \%$ para a CC com a PAS. A CA ainda se associou de maneira positiva e significativa com a LDL e com o HOMA-IR (Tabela 2). Ao ajustar o modelo para o efeito das variáveis indicadoras de gordura total (IMC e \%GC), além da idade, verificase nos meninos que apenas as variáveis CC e RCE mantiveram a associação positiva e significativa com triglicerídeos. O efeito da CC sobre a variação da PAS também se manteve positivo e significativo e independente do percentual de gordura, mas não do IMC. Para meninas quase todas as medidas, com exceção da RCQ, mantiveram a associação positiva com a PAS, independente de \%GC, mas não independente de IMC, assim como se verificou para a CA com o HOMA (Tabela 3).

Tabela 1. Médias expandidas (intervalo de confiança de 95\%) e p-valor das variáveis avaliadas entre estudantes de escolas estaduais de Niterói, RJ, 2003.

\begin{tabular}{lccccc}
\hline \multicolumn{1}{c}{ Variáveis } & \multicolumn{2}{c}{$\begin{array}{c}\text { Masculino } \\
\text { Média (IC 95\%) }\end{array}$} & $\mathbf{n}$ & Meminino \\
\hline Idade & 222 & $16,95(16,40-17,51)$ & 388 & $16,62(16,02-17,21)$ & 0,06 \\
\hline Estatura (cm) & 222 & $174,02(172,53-175,51)$ & 388 & $161,07(159,94-162,21)$ & $<0,00$ \\
\hline IMC $\left(\mathrm{kg} / \mathrm{m}^{2}\right)$ & 222 & $21,16(20,58-21,74)$ & 388 & $21,06(20,55-21,57)$ & 0,77 \\
\hline \%GC & 218 & $15,36(14,13-16,60)$ & 385 & $29,68(28,51-30,86)$ & $<0,00$ \\
\hline CC $(\mathrm{cm})$ & 222 & $72,67(71,50-73,84)$ & 388 & $67,38(66,31-68,46)$ & $<0,00$ \\
\hline RCQ & 222 & $0,78(0,78-0,79)$ & 388 & $0,73(0,72-0,73)$ & $<0,00$ \\
\hline RCE & 222 & $0,42(0,41-0,42)$ & 388 & $0,42(0,41-0,43)$ & 0,89 \\
\hline CA (cm) & 222 & $77,07(75,76-78,38)$ & 388 & $75,79(74,54-77,05)$ & 0,17 \\
\hline Homa-IR & 197 & $1,72(1,19-2,26)$ & 345 & $2,0(1,46-2,53)$ & 0,05 \\
\hline Colesterol total (mg/dl) & 210 & $150,44(142,02-158,86)$ & 367 & $160,45(153,20-167,69)$ & 0,00 \\
\hline LDL-C (mg/dl) & 210 & $87,49(81,21-93,77)$ & 367 & $97,98(91,38-104,59)$ & 0,00 \\
\hline HDL-C (mg/dl) & 210 & $46,37(43,11-49,62)$ & 367 & $46,53(43,53-49,52)$ & 0,91 \\
\hline Triglicerideos (mg/dl) & 210 & $84,37(78,67-90,06)$ & 367 & $79,34(73,38-85,29)$ & 0,16 \\
\hline PAS (mmHg) & 219 & $121,67(118,52-124,82)$ & 387 & $107,69(105,85-109,53)$ & $<0,00$ \\
\hline PAD (mmHg) & 219 & $69,90(67,47-72,32)$ & 387 & $64,77(63,37-66,17)$ & $<0,00$ \\
\hline
\end{tabular}

$\mathrm{IMC}=$ índice de massa corporal; $\mathrm{GC}$ = gordura corporal; $C C=$ circunferência da cintura; $R C Q=$ razão cintura/quadril; $R C E=$ razão cintura/estatura; $C A=$ circunferência abdominal; HOMA-IR = homeostatic model assessment - insulin resistance; LDL-C = low-density lipoprotein cholesterol; HDL-C = high-density lipoprotein cholesterol; PAS = pressão arterial sistólica; PAD = pressão arterial diastólica. 
Tabela 2. Coeficiente de regressão linear $(\beta)$ e $\mathrm{r}^{2}$ para medidas de adiposidade central e as variáveis componentes da SM nos estudantes de escolas estaduais de Niterói, RJ.

\begin{tabular}{|c|c|c|c|c|c|c|c|}
\hline Masculino & & $\mathrm{CC}$ & $\mathrm{RCQ}$ & CA & RCE & IMC & $\% G C$ \\
\hline \multirow[t]{2}{*}{ IMC } & $B$ & 2,00 ** & 0,00 ** & 3,36 ** & 0,00 ** & - & $0,41^{* *}$ \\
\hline & $r^{2}$ & 0,84 & 0,13 & 0,78 & 0,79 & - & 0,61 \\
\hline \multirow[t]{2}{*}{$\%$ GC } & B & $0,78^{* *}$ & 0,00 ** & 0,91 ** & 0,00 ** & 1,50 ** & - \\
\hline & $r^{2}$ & 0,46 & 0,19 & 0,42 & 0,74 & 0,61 & - \\
\hline \multirow[t]{2}{*}{ LDL -C } & B & 0,57 & 11,42 & 0,37 & 8,50 & 0,80 & 0,68 \\
\hline & $r^{2}$ & 0,02 & 0,02 & 0,01 & 0,01 & 0,01 & 0,02 \\
\hline \multirow[t]{2}{*}{ HDL-C } & $B$ & 1,00 & $-0,15$ & 1,00 & $-0,11$ & 1,00 & $-1,00$ \\
\hline & $r^{2}$ & 0,00 & 0,00 & 0,00 & 0,00 & 0,00 & 0,00 \\
\hline \multirow[t]{2}{*}{ TG } & B & 1,02 ** & 0,74 * & 1,01 ** & 1,60 ** & 1,03 * & 1,01 * \\
\hline & $r^{2}$ & 0,10 & 0,04 & 0,07 & 0,08 & 0,06 & 0,06 \\
\hline \multirow[t]{2}{*}{ HOMA-IR } & B & $-1,01$ & $-0,18$ & $-1,00$ & $-0,18$ & $-1,01$ & $-1,01$ \\
\hline & $r^{2}$ & 0,00 & 0,00 & 0,00 & 0,00 & 0,00 & 0,01 \\
\hline \multirow[t]{2}{*}{ PAD } & $B$ & $0,27^{* *}$ & 0,34 & 0,18 * & 2,45 & 0,60 * & 0,22 \\
\hline & $r^{2}$ & 0,03 & 0,00 & 0,18 & 0,00 & 0,03 & 0,02 \\
\hline \multirow[t]{2}{*}{ PAS } & B & $0,82^{* *}$ & 4,84 & 0,61 ** & $11,10^{* *}$ & $1,76^{* *}$ & $0,61^{\text {** }}$ \\
\hline & $r^{2}$ & 0,15 & 0,01 & 0,13 & 0,08 & 0,15 & 0,07 \\
\hline Feminino & & $\mathrm{CC}$ & $\mathrm{RCQ}$ & CA & RCE & IMC & $\%$ GC \\
\hline \multirow[t]{2}{*}{ IMC } & B & 1,92 ** & 0,00 ** & 2,22 ** & 0,00 ** & - & 0,40 ** \\
\hline & $r^{2}$ & 0,85 & 0,23 & 0,78 & 0,84 & - & 0,74 \\
\hline \multirow[t]{2}{*}{$\%$ GC } & B & $0,75^{\text {** }}$ & 0,00 ** & $0,89^{* *}$ & 0,00 ** & 1,83 ** & - \\
\hline & $r^{2}$ & 0,61 & 0,22 & 0,56 & 0,78 & 0,74 & - \\
\hline \multirow[t]{2}{*}{ LDL-C } & B & 0,58 & 0,75 & 0,49 * & $13,15^{*}$ & 1,79 * & $1,15^{* *}$ \\
\hline & $r^{2}$ & 0,01 & 0,00 & 0,02 & 0,04 & 0,04 & 0,07 \\
\hline \multirow[t]{2}{*}{ HDL-C } & B & $-1,00$ & 0,11 & $-1,00$ & 0,10 & 1,00 & 1,00 ** \\
\hline & $r^{2}$ & 0,01 & 0,00 & 0,00 & 0,00 & 0,00 & 0,00 \\
\hline \multirow[t]{2}{*}{ TG } & $B$ & 1,00 & 0,14 & 1,00 & 0,30 & 1,01 & 1,01 ** \\
\hline & $r^{2}$ & 0,01 & 0,00 & 0,00 & 0,02 & 0,02 & 0,01 \\
\hline \multirow[t]{3}{*}{ HOMA-IR } & B & 1,01 & 0,12 & 1,02 * & 0,15 & 1,03 & 1,00 \\
\hline & & 0,22 & 0,93 & 0,03 & 0,82 & 0,21 & 0,78 \\
\hline & $r^{2}$ & 0,01 & 0,00 & 0,02 & 0,00 & 0,02 & 0,00 \\
\hline \multirow[t]{2}{*}{ PAD } & $B$ & 0,38 ** & 2,10 & 0,31 ** & 5,64 ** & 0,86 ** & 0,39 ** \\
\hline & $r^{2}$ & 0,10 & 0,01 & 0,09 & 0,09 & 0,12 & 0,10 \\
\hline \multirow[t]{2}{*}{ PAS } & $B$ & $0,77^{\text {** }}$ & 6,26 ** & $0,61^{* *}$ & $11,13^{* *}$ & 1,61 ** & $0,73^{* *}$ \\
\hline & $r^{2}$ & 0,22 & 0,04 & 0,19 & 0,18 & 0,22 & 0,18 \\
\hline
\end{tabular}

* p valor < 0,05; ** p valor < 0,01. IMC = índice de massa corporal, GC = gordura corporal; $C C=$ circunferência da cintura, $R C Q=$ razão cintura/ quadril, $\mathrm{RCE}$ = razão cintura/estatura; CA = circunferência abdominal; LDL-C = low-density lipoprotein cholesterol; HDL-C = high-density lipoprotein cholesterol; TG = triglicerídeos; HOMA-IR = homeostatic model assessment - insulin resistance; PAS = pressão arterial sistólica; PAD = pressão arterial diastólica. 
Tabela 3. Coeficiente de regressão linear múltipla $(\beta)$ valor de $p$ e $r^{2}$ das medidas de adiposidade central, ajustados por IMC e \%GC, com as variáveis componentes da SM em estudantes de escolas estaduais de Niterói, RJ.

\begin{tabular}{|c|c|c|c|c|c|c|c|c|c|}
\hline \multirow[t]{2}{*}{ Masculino } & & \multicolumn{2}{|c|}{$\mathrm{CC}$} & \multicolumn{2}{|c|}{$\mathrm{RCQ}$} & \multicolumn{2}{|c|}{ CA } & \multicolumn{2}{|c|}{ RCE } \\
\hline & & IMC & $\% G C$ & IMC & $\% G C$ & IMC & $\% G C$ & IMC & $\% G C$ \\
\hline \multirow[t]{2}{*}{ TG } & $B$ & $1,03^{\text {** }}$ & $1,03^{* \star}$ & 0,32 & 0,33 & 1,01 & 0,01 & 2,33 * & 2,12 * \\
\hline & $r^{2}$ & 0,12 & 0,11 & 0,08 & 0,08 & 0,08 & 0,09 & 0,10 & 0,10 \\
\hline \multirow[t]{2}{*}{ PAD } & $B$ & 0,01 & 0,18 & - & - & $-0,02$ & 0,11 & - & - \\
\hline & $r^{2}$ & 0,07 & 0,07 & - & - & 0,07 & 0,07 & - & - \\
\hline \multirow[t]{2}{*}{ PAS } & $B$ & 0,32 & 0,70 ** & - & - & 0,22 & 0,12 & $-9,37$ & $-3,60$ \\
\hline & $r^{2}$ & 0,21 & 0,15 & - & - & 0,21 & 0,07 & 0,22 & 0,07 \\
\hline \multirow[t]{2}{*}{ Feminino } & & \multicolumn{2}{|c|}{$\mathrm{CC}$} & \multicolumn{2}{|c|}{$\mathrm{RCQ}$} & \multicolumn{2}{|c|}{ CA } & \multicolumn{2}{|c|}{ RCE } \\
\hline & & IMC & $\% G C$ & IMC & $\% G C$ & IMC & $\% G C$ & IMC & $\% G C$ \\
\hline \multirow[t]{2}{*}{ LDL-C } & $B$ & - & - & - & - & $-0,54$ & $-0,39$ & 3,66 & 7,06 \\
\hline & $r^{2}$ & - & - & - & - & 0,06 & 0,09 & 0,73 & 0,44 \\
\hline \multirow[t]{2}{*}{ HOMA-IR } & B & - & - & - & - & 1,02 & 1,03 ** & - & - \\
\hline & $r^{2}$ & - & - & - & - & 0,03 & 0,05 & - & - \\
\hline \multirow[t]{2}{*}{ PAD } & $B$ & 0,02 & 0,02 & - & - & 0,05 & 0,16 & $-1,78$ & 0,99 \\
\hline & $r^{2}$ & 0,16 & 0,16 & - & - & 0,16 & 0,16 & 0,16 & 0,15 \\
\hline \multirow[t]{2}{*}{ PAS } & $B$ & 0,41 & 0,68 ** & $-0,23$ & 0,49 & 0,25 & $0,46^{* *}$ & $-0,22$ & 8,51 * \\
\hline & $r^{2}$ & 0,25 & 0,27 & 0,24 & 0,21 & 0,25 & 0,25 & 0,24 & 0,23 \\
\hline
\end{tabular}

*P valor < 0,05; ** $\mathrm{p}$ valor < 0,01. IMC = índice de massa corporal; $G C=$ gordura corporal; $C C=$ circunferência da cintura; $R C Q=$ razão cintura/quadril; $C A=$ circunferência abdominal; $\mathrm{RCE}$ = razão cintura/estatura; $\mathrm{CA}=$ circunferência abdominal; $\mathrm{LDL}-\mathrm{C}=$ low-density lipoprotein cholesterol; TG = triglicerídeos; $\mathrm{HOMA-IR}=$ homeostatic model assessment - insulin resistance; PAS = pressão arterial sistólica; PAD = pressão arterial diastólica.

\section{DISCUSSÃo}

As medidas antropométricas são amplamente utilizadas como indicadoras de localização de gordura central em estudos epidemiológicos que visam a identificar adolescentes com risco para doenças cardiovasculares $(15,24)$. No entanto, ainda não há consenso sobre a melhor medida para este fim, uma vez que um bom indicador de localização deveria associar-se de maneira independente de sexo, idade e adiposidade total com os marcadores de risco para DCV. Por outro lado, a adiposidade é freqüentemente estimada pelo IMC, o qual é muito dependente da idade e do sexo do adolescente (25). Ademais, o efeito preditivo do IMC e da CC para fatores de risco cardiovascular, em crianças e adolescentes do Bogalusa Heart Study, não foi alterado quando avaliado isoladamente ou em conjunto (24).

No presente estudo, a CC foi a melhor medida associada aos componentes da SM, assim como verificado por Lee e cols. (15) em adolescentes americanos brancos e negros, foi considerada um preditor independente do IMC, de resistência à insulina.

A RCQ foi a medida que apresentou a pior associação, não tendo efeito significativo para nenhuma das variáveis investigadas. Em crianças e adolescentes da Nova Zelândia, a CC apresentou melhor associação com a gordura central avaliada por DEXA (dual-energy $x$-ray absorptiometry) do que a RCQ, após ajuste por sexo e idade (26), o que provavelmente explica a sua melhor associação com as alterações metabólicas. O uso 
da RCQ é criticado, por causa da possibilidade dessa razão refletir diferentes componentes da composição corporal, como massa magra, gorda e esquelética (27). Sobretudo nos adolescentes, as rápidas modificações nas proporções da cintura e do quadril, característicos dessa fase, poderiam estar influenciando nos valores encontrados sem, necessariamente, ser reflexo de um aumento de gordura central (12). No entanto, um estudo realizado em adolescentes com sobrepeso no Brasil, verificou que tanto a CC como a $\mathrm{RCQ}$, após ajuste por sexo, maturação sexual e IMC, apresentaram capacidade similar de explicar a variação dos triglicerídeos, enquanto a RCQ ainda explicou melhor a variação do HDL-C e a razão colesterol total/HDL-C (13).

No presente estudo foram encontradas associações das diferentes medidas antropométricas de acúmulo de gordura central com os componentes da SM, que variaram com o gênero. A associação independente de adiposidade total dos triglicerídeos com a CC, verificada nos meninos, já foi evidenciada em indivíduos adultos do sexo masculino (10) e em jovens obesos (13). Essa associação pode estar relacionada ao aumento do tecido adiposo na região abdominal que, favorecendo a resistência à insulina, intensifica a oxidação dos ácidos graxos livres no plasma, fornecendo substrato para a síntese dos triacil glicerol no fígado e aumentando a liberação hepática de VLDL (very low density lipoprotein), rica em triglicerídeos, para o plasma (28). Contudo, não foi encontrada associação da CC com o HOMA-IR que é uma medida indireta de resistência à insulina.

O fato de a associação da CC com os triglicerídeos só ter sido observada nos meninos pode ser em razão do maior acúmulo de gordura central neste grupo, comprovado pela média maior de CC, em comparação com as meninas. Em adultos, foi verificado que para homens e mulheres com valores aumentados de CC, o sexo masculino apresentava maior depósito de gordura visceral, enquanto o feminino, maior acúmulo de tecido abdominal subcutâneo (8). Considerando que a gordura visceral está associada a triglicerídeos elevados, isso pode também explicar as diferenças entre os sexos nas associações encontradas.

A associação com a pressão arterial parece ser muito mais dependente da adiposidade total do que de localização da gordura, uma vez que para as meninas que possuem maior adiposidade do que os meninos e melhor poder de explicação tanto para a PAS quanto para a PAD, o IMC foi um indicador de mesma magnitude da CC. Entretanto, o efeito da CC sobre a PAS foi anulado quando ajustado pelo IMC, em ambos os sexos. Ademais, de todas as variáveis estudadas, a maior capacidade explicativa foi do IMC para a PAS em meninas (22\%).

Dados do Bogalusa Heart Study demonstraram que níveis de pressão arterial mais elevados em crianças, mesmo que dentro dos limites considerados normais, tendem a evoluir ao longo da vida, mantendo a pressão arterial mais elevada e apresentando maior probabilidade de se tornar um adulto hipertenso, sobretudo nos indivíduos obesos (29). Essas evidências comprovam que o sobrepeso é um importante fator associado à hipertensão arterial na adolescência, no entanto, são escassos os estudos que utilizam a CC como medida de associação de risco para hipertensão arterial nessa fase, o que dificulta a comparabilidade dos dados do presente estudo. No Japão, crianças e adolescentes com sobrepeso apresentaram associação significante da gordura visceral, avaliada por método de imagem, com a PAS (30). No Sul do Brasil foi verificado, em adultos, que a CC foi melhor preditora que o IMC para determinar risco para hipertensão arterial (9). Dessa forma, a definição da melhor medida antropométrica associada à pressão arterial ainda carece de mais investigações.

Os resultados do presente estudo confirmam que, assim como nos adultos, a CC isolada parece ser uma medida antropométrica bastante atrativa para identificar adolescentes com risco para a DCV, já que, também nessa fase, se associa a gordura abdominal (26) e a indicadores da SM $(15,24)$.

De um modo geral, os estudos utilizam a recomendação da OMS (31) ou a sugerida pelo Manual de Referência para Padronização de Medidas Antropométricas (21) para aferição da CC, os quais sugerem, respectivamente, o ponto médio entre a última costela e a crista ilíaca e a menor circunferência do tronco. Entretanto, alguns autores usam a denominação e os pontos de corte da circunferência da cintura (waist circumference), mas aferem a medida na cicatriz umbilical $(32,33)$ que, segundo o referido manual, seria a localização para aferição da CA. Esta aparente confusão pode gerar incoerências na interpretação de resultados referentes à utilização das medidas antropométricas de acúmulo de gordura central, na prática clínica.

No estudo, foi utilizada a metodologia do manual (21), na qual a CC é aferida na menor circunferência do tronco e a CA na cicatriz umbilical. A opção pelo uso da medida da CC na menor circunferência foi por causa da facilidade e da praticidade na identificação do local de aferição quando comparada à metodologia da OMS, 
que exige a identificação de marcos anatômicos mais difíceis de serem localizados. Por esta razão, a localização na menor circunferência é freqüentemente utilizada nos estudos epidemiológicos (16). No estudo, foi investigado se as distintas medidas de gordura central tinham associação diferenciada com os componentes da SM. Apesar de o efeito da CA ter sido semelhante ao efeito da CC na associação bivariada (com exceção da associação da CA com valores de HOMA nas meninas), é provável que o uso da CC seja mais apropriado para adolescentes porque, além do desempenho ser igual ou melhor para alguns critérios da SM, esta medida foi recentemente recomendada pelo IDF como componente principal dos critérios para definição da SM em adolescentes, assim como nos adultos (34).

A CC, quando relacionada à estatura (RCE), é considerada um índice importante para monitorar tendência de excesso de peso (5), uma vez que também é altamente correlacionada com gordura visceral (35) e associada a fatores de risco para SM em crianças (36). No presente estudo, a RCE foi a medida de gordura central, depois da CC, que melhor se associou com as variáveis da SM. Particularmente na adolescência, a RCE pode ser importante ferramenta para a avaliação clínica, tendo em vista a possibilidade de demonstrar a relação entre a gordura central com o incremento da estatura.

Embora o estudo transversal não permita a avaliação de causa e efeito em virtude da impossibilidade de avaliar a temporalidade dos eventos, é possível prever, à luz dos conhecimentos advindos dos estudos longitudinais $(37,38)$, que valores aumentados de medidas antropométricas indicadoras de adiposidade antecedam as alterações metabólicas investigadas.

Uma limitação do estudo foi a utilização do manômetro digital para avaliar pressão arterial que, apesar de ser considerado um método aceitável em pediatria (39) e ter sido utilizado, recentemente, para avaliar hipertensão arterial em crianças e adolescentes japoneses (30), pode apresentar limitação, sobretudo, na determinação da pressão diastólica e necessita ser calibrado com mais freqüência (39). Acredita-se, todavia, que esta limitação não tenha alterado significativamente os resultados do estudo, já que foi utilizada a metodologia específica para o aparelho. Outra possível limitação do estudo foi o método de bioimpedância utilizado para avaliação do \%GC, pelo sistema membro inferior a membro inferior que, provavelmente, apresenta menor poder para estimar gordura abdominal e, portanto, não estimando corretamente a adiposidade total, o que talvez possa explicar a associação da CC com a PAS, independente de $\% \mathrm{GC}$, mas não de IMC. Finalmente, não foram utilizados dados de maturação sexual, por causa das dificuldades operacionais para esta avaliação, sobretudo em estudos epidemiológicos, embora reconhecida a importância desta informação para a interpretação do desenvolvimento na adolescência, expresso nas medidas antropométricas.

Conclui-se que a CC foi a melhor medida de localização de gordura central associada aos fatores de risco para DCV nos adolescentes avaliados, particularmente para triglicerídeos, evidenciando-se a importância da aferição desta medida na prática clínica e em estudos de triagem de adolescentes em risco. Contudo, considerando que, de modo geral, os efeitos da CC foram atenuados quando ajustados para o IMC, é provável que nos adolescentes estudados, por apresentarem ainda menor adiposidade total (40) do que, por exemplo, adolescentes americanos, a localização de gordura na região central possa ser ainda pequena e, causar impacto mais reduzido nos fatores de risco cardiovascular.

\section{AGRADECIMENTOS}

Ao Conselho Nacional de Desenvolvimento Científico e Tecnológico (CNPq) que financiou este estudo (Processo $\left.\mathrm{n}^{\circ} 505.524 / 2004-0\right)$.

\section{REFERÊNCIAS}

1. Cook S, Weitzman M, Auinger P, Nguyen M, Dietz WH. Prevalence of a metabolic syndrome phenotype in adolescents. Findings from the third National Health and Nutrition Examination Survey, 1988-1994. Arch Pediatr Adolesc Med. 2003;157: 821-7.

2. Alvarez MM, Vieira ACR, Moura AS, Veiga GV. Insulin resistance in Brazilian adolescent girls: association with overweight and metabolic disorders. Diab Res Clin Pract. 2006;74:183-8.

3. Silva RCQ, Miranda WL, Chacra AR, Dib AS. Metabolic syndrome and insulin resistance in normal glucose tolerant Brazilian adolescents with family history of type 2 diabetes. Diabetes Care. 2005;28-716-8.

4. Li C, Ford ES, Mokdad AH, Cook S. Recent trends in waist circumference and waist-height ratio among US children and adolescents. Pediatrics. 2006;118:1390-8.

5. van der KK, Seidell JC. Techniques for the measurement of visceral fat: a pratical guide. Int J Obes Relat Metab Disord. 1993;17:187-96.

6. Alberti KG, Zimmet $P$, Shaw J. IDF epidemiology task force consensus group. The metabolic syndrome - a new worldwide definition. Lancet. 2005;366:1059-62. 
7. Alberti KGMM, Zimmet PZ. Definition, diagnosis and classification of diabetes mellitus and its complications. Part 1: diagnosis and classification of diabetes mellitus, provisional report of a WHO consultation. Diabetic Med. 1998;15:539-53.

8. Kuk JL, Lee S, Heymsfield SB, Ross R. Waist circumference and abdominal adipose tissue distribuition: influence of age and sex. Am J Clin Nutr. 2005;81:1330-4.

9. Gus M, Fuchs SC, Moreira LB, Moraes RS, Wiehe M, Silva AF, et al. Association between different measurements of obesity and the incidence of hypertension. Am J Hypertens. 2004;17:50-3.

10. Lemos-Santos MGF, Valente JG, Gonçalves-Silva RMV, Sichieri $\mathrm{R}$. Waist circumference and waist-to-hip ratio as predictors of serum concentration of lipids in Brazilian men. Nutrition. 2004;20:857-62.

11. Pereira RA, Sichieri R, Marins V. Razão cintura/quadril como preditor de hipertensão arterial. Cad Saude Publica. 1999;15:333-44.

12. Weiss R, Dziura J, Burgert T, Tamborlane WV, Taksali SE, Yeckel CW, et al. Obesity and the metabolic syndrome in children and adolescents. N England J Med. 2004;350:2362-74.

13. Oliveira CL, Veiga GV, Sichieri R. Anthropometric markers for cardiovascular disease risk factors among overweight adolescents. Nutr Res. 2001;21:1335-45.

14. Gillum RF. Distribuition of waist-to-hip ratio, other indexes of body fat distribuition and obesity and associations with HDL cholesterol in children and young adults aged 4-19 y: the Third National Health and Nutrition Examination Survey. Int J Obes Relat Metab Disord. 1999;23:556-63.

15. Lee S, Bacha F, Gungor N, Arslanian SA. Waist circunference is an independent predictor of insulin resistance in black and white youths. J Pediatr. 2006;148:188-94.

16. Wang J, Thornnton JC, Bari S, Williamson B, Gallagher D, Heymsfield SB, et al. Comparisons of waist circumference measured at 4 sites. Am J Clin Nutr. 2003;77:379-84.

17. Gerber IRS, Zielinsky P. Fatores de risco de aterosclerose na infância, um estudo epidemiológico. Arq Bras Cardiol. 1997;69:231-6.

18. Lwanga SK, Lemeshow S. Sample size determination in health studies: a pratical manual. World Health Organization: Geneva, 1991.

19. Gordon CC, Chumlea WC, Roche AF. Estature, recumbent, lenght, and weight. In: Lohman TG, Roche AF, Martorell R. Antropometric standardization reference manual. Champaign, Illinois: Human Kinetics Books; 1988.

20. Boileau RA. Body composition assessment in children and youth. In: Bar-Or O, editor. The child and adolescent athlete. Oxford: Blackwell Science; 1996.

21. Callaway $\mathrm{CN}$, Chumlea WC, Bouchard C, Himes JH, Lohman TG, Martim AD, et al. Antropometric standardization reference manual. Champaign, Illinois: Human Kinetics Books; 1988.

22. Friedwald WT, Levy RI, Fredrickson. Estimation of the concentration of low-density lipoprotein cholesterol in plasma, without use of the preparative ultracentrifuge. Clin Chem. 1972;499:502.

23. Matthews DR, Hosker JP, Rudenski AS, Naylor BA, Treacher DF, Turner RC. Homeostasis model assessment: insulin resistance and ß-cell function from fasting plasma glucose and insulin concentrations in man. Diabetologia. 1985;28:412-9.

24. Janssen I, Katzmarzyk PT, Srinivasan SR, Chen W, Malina RM, Bouchard $C$, et al. Combined influence of body mass index and waist circunference on coronary artery risk factors among children and adolescents. Pediatrics. 2005;115:1623-30.
25. Cole JT, Bellizi CM, Flegal MK, Dietzs HW. Establishing a standard definition for child overweight and obesity worldwide: international survey. BMJ. 2000;320:1240-3.

26. Taylor RW, Jones IE, Williams SM, Goulding A. Evaluation of waist circumference, waist-to-hip ratio, and the conicity index as screening tools for high trunk fat mass, as measured by dual-energy $X$-ray absorptiometry, in children aged 3-19 y. Am J Clin Nutr. 2000;72:490-5.

27. Mollarius A, Seidell JC. Selection of anthropometric indicators for classification of abdominal fatness - a critical review. Int J Obes Relat Metab Disord. 1998;22:719-27.

28. Ruan H, Lodish HF. Insulin resistance in adipose tissue: direct and indirect effects of tumor necrosis factor- . Cytokine Growth Factor Rev. 2003;14:447-55.

29. Kay JD, Sanaiko AE, Stephen RD. Pediatr hypertension. Am Heart J. 2001;143:422-32.

30. Nishina M, Kikuchi T, Yamazaki H, Kameda K, Hiura M, Uchiyama M. Relationship among systole blood pressure, serum insulin and leptin, and visceral fat accumulation in obese children. Hypertens Res. 2003;26:281-8.

31. Lean MEJ, Han TS, Deurenberg P. Predicting body composition by densitometry from simple anthropometric measurements. Am J Clin Nutr. 1996;63:4-14.

32. Ribeiro AB, Gimeno SGA, Andreoni S, Ferreira SRG. Japanese Brazilian diabetes group. Should body mass index be adjusted for relative sitting height in cross-sectional studies of chronic diseases in Japanese-Brazilians? Cad Saude Publica. 2006;22:1691-7.

33. Daniels SR, Khoury PR, Morrinson JA. Utility of different measures of body faty distribuition in children and adolescents. Am J Epidemiol. 2000;152:1179-84.

34. Alberti KGMM, Zimmet PZ, Shaw JE. The metabolic syndrome in children and adolescents. Lancet. 2007;369:2059-61.

35. Ashwell M, Cole TJ, Dixon AK. Ratio of waist circumference to height is strong predictor of intra-abdominal fat. BMJ. 1996;313:559-60.

36. Savva SC, Tornaritis M, Savva ME. Waist circumference and waist-to-weight ratio are bether predictors of cardiovascular disease risk factors in children than body mass index. Int $\mathrm{J}$ Obes Relat Metab Disord. 2000;24:1453-8.

37. Tershakovec AM, Jawad AD, Stouffer O, Elkasabany A, Scrinivasan $\mathrm{R}$, Berenson GS. Persistent hypercholesterolemia is associated with the development of obesity among girls: the Bogalusa Heart Study. AJCN. 2002;76:730-5.

38. Valente AM, Strong W, Sinaiko AR. Obesity and insulin resistance in young people. Am Heart J. 2001;142:440-4.

39. Salgado MC, Carvalhães JTA. Arterial hypertension in childhood. J Pediatr (Rio J). 2003;79 Suppl. 1:S115-24.

40. Vieira ACR, Alvarez MM, Marins VMR, Sichieri R, Veiga GV. Desempenho de pontos de corte do índice de massa corporal de diferentes referências na predição de gordura corporal em adolescentes. Cad Saude Publica. 2006;22:1681-90.

\section{Endereço para correspondência:}

Marlene Merino Alvarez

Instituto de Nutrição Josué de Castro - UFRJ

Av. Brigadeiro Trompowisky s $/ \mathrm{n}^{\circ}$ - Centro de Ciências da

Saúde - Bloco J - $2^{\circ}$ andar, llha do Fundão

21941-590, Rio de Janeiro, RJ

E-mail:marlene.merino@globo.com 